\title{
Therapy refractory hypertension in adults: aortic coarctation has to be ruled out
}

\author{
M. E. W. Hemels • E. S. Hoendermis • J. P. van Melle • \\ P. G. Pieper
}

Published online: 11 February 2011

(C) The Author(s) 2011. This article is published with open access at Springerlink.com

\begin{abstract}
In patients with unexplained hypertension, especially in combination with a cardiac murmur, the presence of an aortic coarctation should always be ruled out given the high morbidity and mortality. However, particularly patients with an isolated coarctation often remain asymptomatic for years and the defect may be unnoticed even until the fifth or sixth decade of life. In the present article, we describe two patients with late detected coarctation to illustrate the clinical consequences, diagnostic clues for earlier detection and current therapeutic options to achieve optimal treatment. The key sign of an aortic coarctation, a difference in arterial blood pressure measured between the upper and lower extremities, should always be examined, followed by echocardiography. We conclude that even in case of a late detected severe coarctation, surgical or percutaneous repair has proven to be feasible and substantially effective, improving quality of life and lowering the risk of further hypertension-associated problems.
\end{abstract}

Keywords Hypertension - Aortic coarctation - Adult .

Diagnosis and treatment

\section{Introduction}

Coarctation of the aorta is a localised stenosis of the aorta, most commonly located just distal to the origin of

M. E. W. Hemels $(\varangle) \cdot$ E. S. Hoendermis $\cdot$ J. P. van Melle

P. G. Pieper

Department of Cardiology, Thoraxcenter,

University Medical Center Groningen, University of Groningen,

PO Box 30.001,

9700 RB Groningen, the Netherlands

e-mail: m.e.w.hemels@thorax.umcg.nl the left subclavian artery and closely related to the attachment of the ductus arteriosus with the aorta. It accounts for $5 \%$ to $9 \%$ of all congenital cardiovascular defects [1]. Frequently associated congenital defects are a bicuspid aortic valve, valvular and subvalvular aortic stenosis, mitral stenosis, ventricular septal defect and persistent ductus arteriosus [2]. Coarctation causes an increased afterload for the left ventricle, resulting in an increased systolic pressure in the left ventricle and upper part of the body. Treatment of aortic coarctation can be performed surgically or percutaneously. Even after successful correction hypertension often persists due to abnormal vascular physiology. Uncorrected or unnoticed coarctation results in a high morbidity and mortality, mainly due to hypertension-associated problems [3]. However, patients with isolated coarctation (coarctation without additional congenital defects) often remain asymptomatic for years and the defect may be unnoticed even until the fifth or sixth decade of life. Although a late detected coarctation almost always includes irreversible cardiovascular damage, adequate treatment can result in a substantial improvement in both quality of life and prognosis [4-7]. In the present article, we describe two patients with late detected coarctation to illustrate the clinical consequences, diagnostic clues for earlier detection and current therapeutic options to achieve optimal treatment in these patients.

\section{Case A}

A 40-year-old woman was referred to our hospital in 2004 because of a recently discovered difference in blood pressure between the right and left arm and a cardiac murmur. Hypertension had already been diagnosed 20 years 
before and she was treated with an angiotensin receptor blocker. Every now and then she suffered from chest pain, especially in bed at night, sporadically during exertion. She had no pregnancy wish and besides hypertension and migraine she had no further medical history. The arterial blood pressure at her right arm was $155 / 85 \mathrm{mmHg}$ and on the left arm 135/70 $\mathrm{mmHg}$, arterial blood pressure was unobtainable in the lower extremities, and there was a marked delay in the femoral pulses compared with the brachial arteries ('femoral-brachial delay'). Auscultation of the chest revealed an early systolic ejection murmur and a soft diastolic murmur. The systolic murmur was also heard on the posterior thorax. The electrocardiogram showed normal sinus rhythm and conduction times, but also signs of left ventricular hypertrophy (high R-waves in the left precordial leads with associated ST-segment changes). At transthoracic echocardiography, left and right ventricular dimensions and function appeared within normal ranges. However, a functional bicuspid aortic valve was identified, with a grade I/III regurgitation. The diameter of the ascending aorta was enlarged $(38 \mathrm{~mm})$. Suprasternal view revealed poor acoustic images, but in the abdominal aorta a clear diastolic forward flow was demonstrated. Aortic coarctation was suspected and confirmed by magnetic resonance (MR) imaging showing a wide aortic arch and a significant narrowing, with minimal diameter of $6 \mathrm{~mm}$, just distal of the left subclavian artery and poststenotic dilatation of the descending aorta. The systolic blood pressure gradient between upper and lower extremities appeared to be $30-40 \mathrm{mmHg}$. An uncomplicated balloon angioplasty was performed (using a $30 \times 14$ and $40 \times 18 \mathrm{~mm}$ balloon, respectively) resulting in a coarctation diameter of $16 \mathrm{~mm}$ (Fig. 1a and b). A stent was not placed because of the existence of wide pre- and post-coarctation diameters. Given the elastic aortic properties, solid stent fixation was not expected. During follow-up of 5 years, no recoarctation occurred, the systolic blood pressure gradient between upper and lower extremities remained $10 \mathrm{mmHg}$ and blood pressure control was satisfactory using low doses of atenolol and losartan.

\section{Case B}

A 55-year-old woman was seen at the outpatient clinic in 2007 because of progressive complaints of angina. Her medical history included hypertension and a cardiac murmur, which had already been diagnosed during a medical examination when she was 18 years old. In 1991, she was evaluated because of symptoms of dizziness and near collapse during exertion. Besides systolic hypertension, no structural heart disease was found except for a mild aortic regurgitation and a peak systolic pressure gradient of $17 \mathrm{mmHg}$ (heart rate 68 beats/min) over the aortic valve. She was treated with antihypertensive drugs and visited her cardiologist on a regular, yearly basis. Because of therapy refractory hypertension she was also referred to the internist to exclude underlying causes of her hypertension, but no underlying cause was diagnosed. When she was 50 years old, she stopped smoking because mild chronic obstructive pulmonary disease was diagnosed. At that time echocardiography showed left ventricular hypertrophy (septum en posterior wall diameter both $12 \mathrm{~mm}$ ) with normal systolic function, still a mild aortic regurgitation and a peak systolic pressure gradient across the aortic valve of $28 \mathrm{mmHg}$ (heart rate 64 beats $/ \mathrm{min}$ ). In the meantime, she was treated with four different antihypertensive drugs, but she remained hypertensive. To evaluate her complaints of angina in 2007 a nuclear exercise stress test was performed, which showed signs of reversible perfusion defects in the inferior, posterior and lateral walls suggestive of myocardial ischaemia. During following cardiac catheterisation, via the right femoral artery, the catheter could not be advanced up the ascending aorta because of a discrete narrowing of the aorta (Fig. 2). Catheterisation performed via the radial artery showed a significant stenosis in the circumflex artery
Fig. 1 a Aortic coarctation (red line: $6 \mathrm{~mm}$ ) distal to the left subclavian artery, before balloon angioplasty; b aortic coarctation (red line: $16 \mathrm{~mm}$ ), after balloon angioplasty
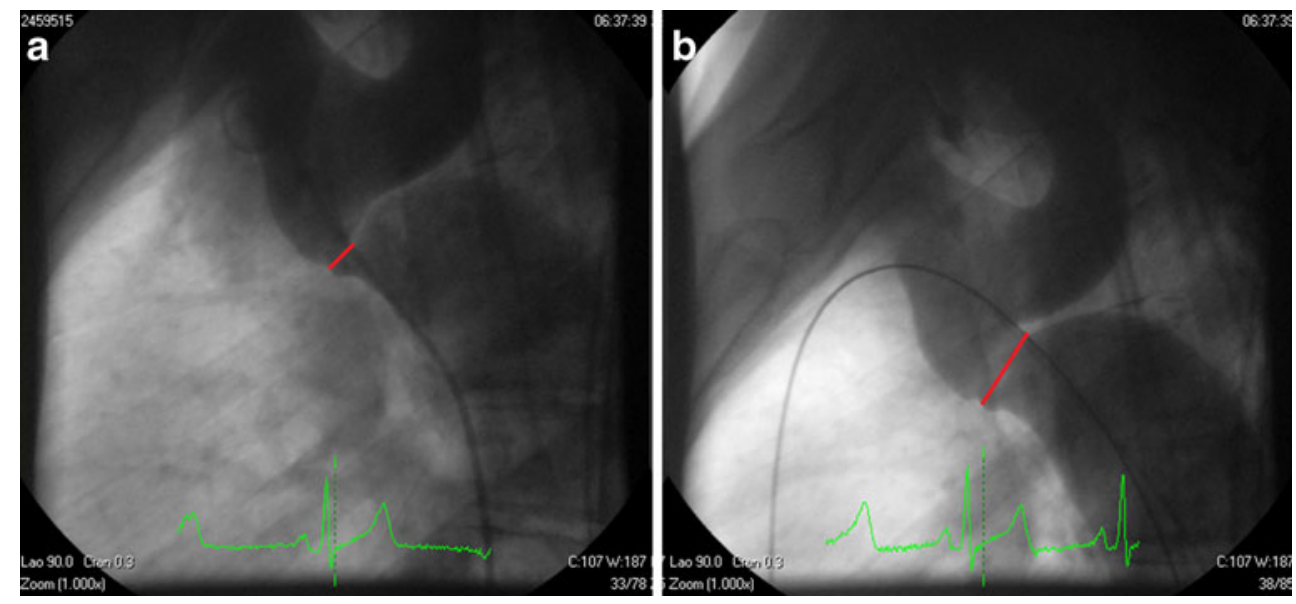
which was subsequently dilated and stented. The calculated peak pressure gradient across the narrowed thoracic aorta was $30 \mathrm{mmHg}$. Additional computed tomography (CT) scan of the aorta confirmed the diagnosis of coarctation with a pinpoint stenosis in the aorta just distal to the left subclavian artery. Moreover, an extensive collateral network, mainly consisting of bronchial and lumbar arteries, was seen (Fig. 3). Atherosclerotic changes in the cerebral vasculature were excluded by a digital subtraction angiography. It was decided by our congenital team to accomplish an extra-anatomical bypass 1 year after coronary stenting (Fig. 4). Via a posterolateral thoracotomy an extraanatomical bypass was constructed between the aortic isthmus and descending aorta by the use of a 16-mm prosthetic graft. No perioperative complications occurred and postoperatively, for the first time in her life, her blood pressure was better under control with antihypertensive drugs even with dose reduction of the medication. After more than 2 years of follow-up she was still free of angina, experienced a better exercise tolerance and her blood pressure levels were under control.

\section{Discussion}

We have described two women with a late diagnosed isolated coarctation, although hypertension had been diagnosed decades before. Paradoxically, isolated aortic coarctation has a male to female predominance [8]. The most common type of 'asymptomatic' coarctation is a localised, short segment, narrowing near the ligamentum arteriosum. It is frequently accompanied by a bicuspid aortic valve (60-80\%) [9]. In retrospect, in both cases, hypertension in combination with a cardiac murmur was already noticed during early adulthood which should have raised the physicians suspicion. In addition, other diagnostic clues

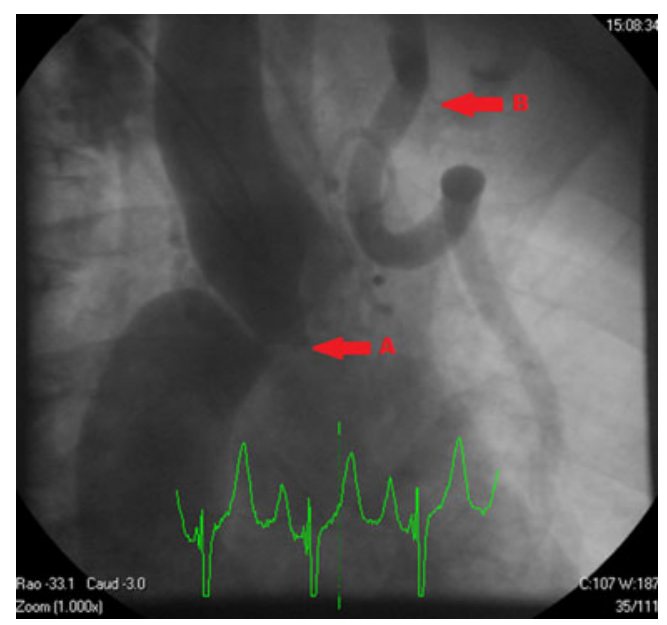

Fig. 2 Pinpoint aortic coarctation $(A)$ and collateral vessel $(B)$

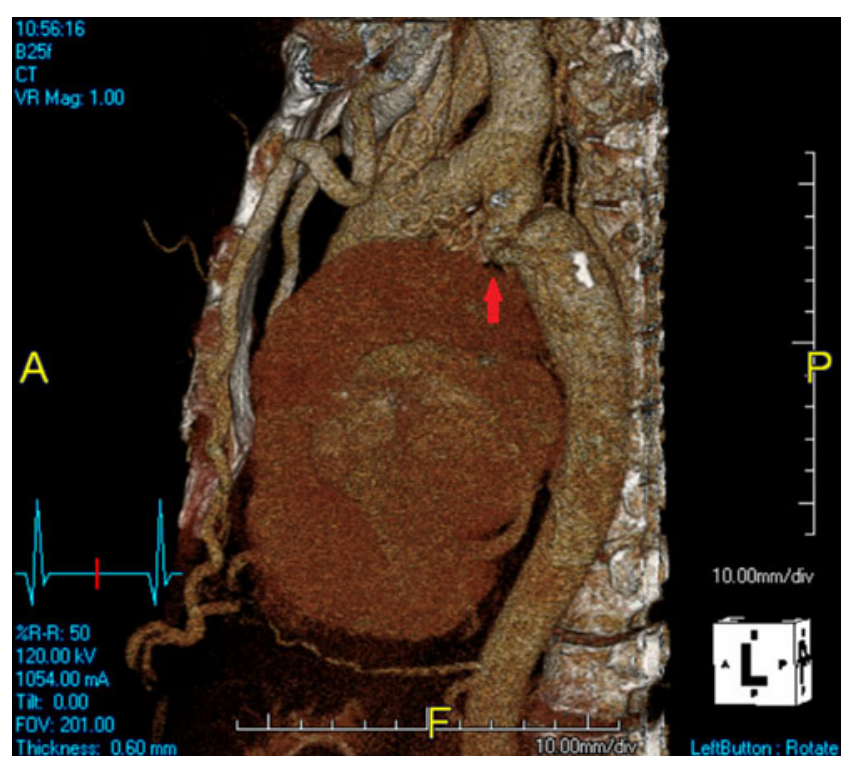

Fig. 3 CT image of severe aortic coarctation (red arrow) just distal to the left subclavian artery, and an extensive collateral network
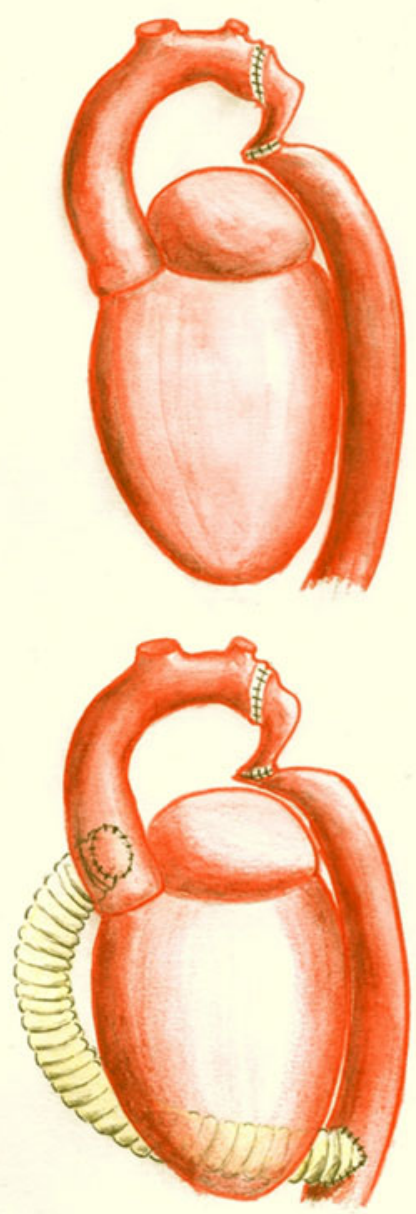

Fig. 4 Extra-anatomical bypass of aortic coarctation 
were brachial-femoral delay (delayed femoral pulses), weak femoral pulsations, arterial blood pressure difference between the right and left arm as well as between upper and lower extremities and finally auscultation on the posterior thorax (continuous murmur caused by collateral vessels or systolic murmurs due to coarctation), see also Table 1 . From a clinician's standpoint it is important to realise that the absence of a difference in blood pressure between the upper extremities does not exclude the presence of aortic coarctation, because in the majority of cases the coarctation is localised distal to the origin of the left subclavian artery. The key sign of an aortic coarctation is therefore a difference in arterial blood pressure measured between the upper and lower extremities. This examination should always be performed in case of unexplained hypertension in (young) adults. The electrocardiogram may show signs of left ventricular hypertrophy due to longstanding hypertension. Specific signs on a chest X-ray are inferior rib notching due to enlarged and tortuous intercostal arteries (in the posterior-anterior view), and aortic contour abnormalities (on the lateral view). In addition, directed (colour) Doppler echocardiography can confirm the diagnosis or make it less likely, and is important to screen for additional cardiac defects. In the suprasternal long-axis view discrete narrowing of the thoracic aorta may be visualised. Furthermore, diastolic forward flow can be identified in the descending thoracic aorta and/or in the abdominal aorta which is specific for an aortic coarctation (Fig. 5). The severity of the stenosis can be estimated by measuring maximal flow velocity in the descending aorta as compared with prestenosis flow velocity. Calculation of the gradient by the simplified Bernoulli's equation: pressure $=4 \times$ (velocity) [2] should be used with care because the formula is not applicable in non-localised, elongated stenosis. When a coarctation is diagnosed or suspected, imaging by MR of CT can clearly define the localisation of the coarctation, gives a complete view of the aorta including collateral vessels, and can be used to exclude cerebral aneurysms. Additional cardiac catheterisation can help to determine the severity of the stenosis and is indicated when associated coronary artery disease is suspected.

A severe aortic coarctation detected during (early) childhood or in young adults needs to be repaired [10, 11]. Until recently, however, there was no consensus regarding the optimal treatment strategy for late detected

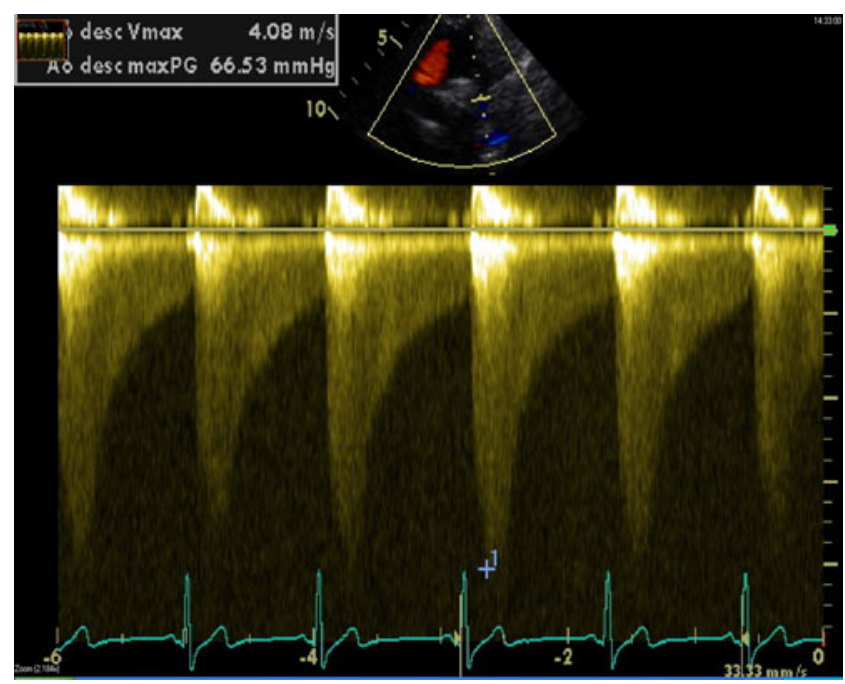

Fig. 5 Echo Doppler image of typical 'sawtooth' with diastolic forward flow in descending aorta, in a patient with severe aortic coarctation

aortic coarctation, because the benefits of surgery were unclear. For example, the regression of systolic hypertension after repair has been seriously questioned [12]. In addition, expected surgical risks in these patients are higher as compared with surgery during childhood $[12,13]$. Yet, the main concern was that a late detected coarctation has already resulted in substantial degenerative changes in the aorta and the presence of an extensive network of collateral vessels resulting in an increased perioperative risk including bleeding, stroke and, although rare, paraplegia especially in case of a poor collateral circulation. Moreover, preoperative coexisting coronary artery disease, left ventricular dysfunction and cerebral vascular disease increase the risk of perioperative complications. However, in the last 10 years several studies have showed the feasibility and benefits of surgical repair in patients older than 50 years $[4-7,14,15]$. The reported perioperative and early mortality in these studies was $0 \%$ and the late mortality $0-4 \%$ (mean followup $0.1-4.3$ years). Perioperative complications occurred in $0-25 \%$ of patients including postperfusion lung oedema, significant pleural effusion, re-exploration for control of bleeding and persistent left vocal cord paralysis.

Rebound hypertension is a well-known phenomenon early after repair of a coarctation. However, the majority of studies have also demonstrated a substantial improvement

Table 1 Physical diagnostic clues for aortic coarctation

\footnotetext{
Hypertension in combination with a cardiac murmur

Difference in arterial blood pressure between upper and lower extremities (and between right and left arm)

Brachial-femoral delay (delayed femoral pulses)

Weak femoral pulsations

Continuous murmur during auscultation on the posterior thorax
} 
in the control of systolic hypertension and symptoms during follow-up, even in patients with mild preoperative hypertension $[5-7,14,16]$. These results are of overriding importance in the decision to repair late detected aortic coarctation, preventing hypertension-related complications in the further course of the patient's life.

The most applied surgical method in patients with a late detected coarctation is an extra-anatomical bypass (Fig. 4). Perioperative surgical morbidity or mortality may occur but is rare [11]. In recent years, a less invasive alternative for surgery, balloon angioplasty with or without stenting of the aorta, has increasingly been used and shows promising results $[17,18]$. The technique proved to be feasible, and the rate of recoarctation appeared to be low, similar to that of surgical methods [18, 19]. Disadvantages of this technique include a per procedure risk of aortic dissection, immediate residual pressure gradients, stent migration and the risk (comparable with surgical repair) of aortic aneurysms during follow-up [20]. The final choice of treatment for late detected aortic coarctation depends upon several factors including the morphology and severity of the coarctation, vascular condition including collateral vessels and comorbidity, and should always be determined in a specialised centre by a team of cardiologists and surgeons with experience in adult congenital heart disease [11].

\section{Conclusion}

In patients with unexplained hypertension, especially in combination with a cardiac murmur, the presence of an aortic coarctation should always be ruled out given the high morbidity and mortality. The key sign of an aortic coarctation, a difference in arterial blood pressure measured between the upper and lower extremities, should always be examined, followed by echocardiography. Even in case of a late detected severe coarctation, surgical or percutaneous repair has proven to be feasible and substantially effective, improving quality of life and lowering the risk of further problems associated with hypertension. Finally, aortic coarctation should be regarded as a diffuse arteriopathy and therefore evaluation and treatment in a specialised centre remains essential aiming for an optimal treatment strategy.

\section{Disclosures None.}

\section{Conflicts of interest None.}

Open Access This article is distributed under the terms of the Creative Commons Attribution Noncommercial License which permits any noncommercial use, distribution, and reproduction in any medium, provided the original author(s) and source are credited.

\section{References}

1. Fyler DC, Buckley LP, Hellenbrand WE. Report of the New England regional infant cardiac program. Pediatrics. 1980;65:432-6.

2. Levine JC, Sanders SP, Colan SD, et al. The risk of having additional obstructive lesions in neonatal coarctation of the aorta. Cardiol Young. 2001;11:44-53.

3. Campbell M. Natural history of coarctation of the aorta. Br Heart J. 1970;32:633-40.

4. Aris A, Subirana MT, Ferres P, et al. Repair of aortic coarctation in patients more than 50 years of age. Ann Thorac Surg. 1999;67:1376-9.

5. Bauer M, Alexi-Meskishvili VV, Bauer U, et al. Benefits of surgical repair of coarctation of the aorta in patients older than 50 years. Ann Thorac Surg. 2001;72:2060-4.

6. Bryant III R, Gonzalez-Stawinski G, Pettersson GB, et al. Surgical management of coarctation of the aorta in adults with concurrent cardiac and aortic disease. J Card Surg. 2008;23:787-90.

7. Vohra HA, Adamson L, Haw MP. Does surgical correction of coarctation of the aorta in adults reduce established hypertension? Interact Cardiovasc Thorac Surg. 2009;8:123-7.

8. Samanek M. Boy:girl ratio in children born with different forms of cardiac malformation: a population-based study. Pediatr Cardiol. 1994;15:53-7.

9. Bjork VO, Bergdahl L, Jonasson R. Coarctation of the aorta. The world's longest follow-up. Adv Cardiol. 1978;22:205-15.

10. Carr JA, Amato JJ, Higgins RS. Long-term results of surgical coarctectomy in the adolescent and young adult with 18-year follow-up. Ann Thorac Surg. 2005;79:1950-5.

11. Warnes CA, Williams RG, Bashore TM, et al. ACC/AHA 2008 guidelines for the management of adults with congenital heart disease: a report of the American College of Cardiology/American Heart Association Task Force on Practice Guidelines (writing committee to develop guidelines on the management of adults with congenital heart disease). Circulation. 2008;118:e714-833.

12. Kaemmerer H, Oelert F, Bahlmann J, et al. Arterial hypertension in adults after surgical treatment of aortic coarctation. Thorac Cardiovasc Surg. 1998;46:121-5.

13. Brouwer RM, Erasmus ME, Ebels T, et al. Influence of age on survival, late hypertension, and recoarctation in elective aortic coarctation repair. Including long-term results after elective aortic coarctation repair with a follow-up from 25 to 44 years. J Thorac Cardiovasc Surg. 1994;108:525-31.

14. Wells WJ, Prendergast TW, Berdjis F, et al. Repair of coarctation of the aorta in adults: the fate of systolic hypertension. Ann Thorac Surg. 1996;61:1168-71.

15. Connolly HM, Schaff HV, Izhar U, et al. Posterior pericardial ascending-to-descending aortic bypass: an alternative surgical approach for complex coarctation of the aorta. Circulation. 2001;104 Suppl 1:I133-7.

16. Vriend JW, van Montfrans GA, Romkes HH, et al. Relation between exercise-induced hypertension and sustained hypertension in adult patients after successful repair of aortic coarctation. J Hypertens. 2004;22:501-9.

17. Zabal C, Attie F, Rosas M, et al. The adult patient with native coarctation of the aorta: balloon angioplasty or primary stenting? Heart. 2003;89:77-83.

18. Chessa M, Carrozza M, Butera G, et al. Results and mid-long-term follow-up of stent implantation for native and recurrent coarctation of the aorta. Eur Heart J. 2005;26:2728-32.

19. Saba SE, Nimri M, Shamaileh Q, et al. Balloon coarctation angioplasty: follow-up of 103 patients. J Invasive Cardiol. 2000;12:402-6.

20. Fawzy ME, Awad M, Hassan W, et al. Long-term outcome (up to 15 years) of balloon angioplasty of discrete native coarctation of the aorta in adolescents and adults. J Am Coll Cardiol. 2004;43:1062-7. 